\title{
A preliminary investigation into the use of alginates for the lifting and enhancement of fingermarks in blood
}

\author{
Mhairi Munro $^{1}$, Paul Deacon ${ }^{2}$, Kevin J. Farrugia ${ }^{1} *$ \\ ${ }^{1}$ School of Science, Engineering \& Technology, Division of Computing and Forensic \\ Sciences, University of Abertay, Dundee, DD1 1HG, UK \\ ${ }^{2}$ c/o School of Science, Engineering \& Technology, Division of Computing and Forensic \\ Sciences, University of Abertay, Dundee, DD1 1HG, UK
}

* Corresponding Author:

School of Science, Engineering \& Technology

Division of Computing and Forensic Sciences

University of Abertay

Dundee DD1 1HG

United Kingdom

tel: $+44(0) 1382308689$

kevin.farrugia@abertay.ac.uk 


\begin{abstract}
Recent studies have reported the use of alginate in the lifting and subsequent enhancement of footwear marks in blood. A study was set up to assess the use of such a method in the treatment of fingermarks in blood on a variety of porous, non-porous and semi-porous surfaces. Other variables included ageing of the fingermarks in blood and the application of chemicals prior to or post-alginate lifting. All different variations were compared to direct chemical treatment of the substrate. The results demonstrated that alginate is not compatible with certain substrates (e.g. glass and tile). On substrates that were compatible with alginate (e.g. fabric and paper), the enhanced fingermarks on the alginate cast and the enhanced fingermarks on the post-alginate substrates appeared, overall, inferior compared to direct chemical enhancement without the use of alginate. A further variation using water-based protein stains directly mixed with the alginate appeared to provide enhancement directly on the substrate as well as simultaneously lifting and enhancing the fingermarks in blood on the alginate cast.
\end{abstract}

Keywords: fingermarks, alginate, blood enhancement, acid black 1, acid yellow 7, leuco crystal violet 


\section{INTRODUCTION}

Alginate casting is commonly used in the field of dentistry for casting a patient's mouth and/or jaw [1]. Alginate is generally supplied as a powder, to which water is added and mixed before application and setting. The typical composition of the powder is $11-16 \%$ sodium or potassium salts of alginic acid (originates from brown seaweed and is the main reactive ingredient that forms a solution with water to form a gel), 11-17\% gypsum $\left(\mathrm{CaSO}_{4} \cdot 2 \mathrm{H}_{2} \mathrm{O}\right.$ - provides the $\mathrm{Ca}^{2+}$ ions that cross-link the alginate chains), $1-3 \%$ trisodium phosphate (controls the setting time), and 65-75\% inert fillers (allows for easy manipulation) [2,3]. Research [3-5] has demonstrated that alginate provides a good medium for lifting blood marks since it binds permanently to the blood to retain most of the detail. A proposed mechanism is that the blood is partially solubilised by the water in the alginate mixture upon application, and then retained when the cast sets [3]. Other similar substances (e.g. dental stone, Mikrosil ${ }^{\circledR}$ and polyvinylsiloxane) have been investigated to assess if they have similar capabilities; however, alginate was the only suitable substance for the lifting of blood marks from the substrates tested (concrete, fabric and human skin) [4]. A major drawback of alginate casting is that the casts shrink quickly over time and therefore immediate photography is recommended after enhancement. Several studies $[3,5,6]$ have compared a range of alginate brands and all concluded that GC Aroma Fine Dust III gave superior results. Alginate has proven to be unreactive with blood enhancement techniques such as leuco crystal violet (LCV) and acid black 1 (AB1), allowing chemical enhancement of the lifted blood mark on the alginate $[4,5]$. In addition, the original blood marks appear to remain intact and largely unchanged, which shows the alginate's non-destructive nature although other studies demonstrated that subsequent enhancement of footwear marks directly on fabric items after alginate lifting was not successful [6]. Two recent studies [3, 6] are in agreement that acid black 1 (AB1) provides superior enhancement of the blood marks lifted on the alginate cast when compared to LCV whilst DFO did not provide any enhancement.

The aim of this study was to assess the capability of GC Aroma Fine Dust III alginate in the lifting and subsequent enhancement of fingermarks in blood (in situ and on alginate cast) on a variety of porous, non-porous and semi-porous surfaces. 


\section{MATERIALS AND METHODS}

The initial substrates selected for this study included glass, tiles, leaflets, white paper, dark denim and black polycotton. The alginate selected for this study is GC Aroma Dust Fine III. The fingermarks in blood were lifted with alginate casting and a comparison of two enhancement techniques were carried out on the alginate casts. The fingermarks in blood on the original surface post-alginate lifting were then treated with one of three enhancement techniques depending on the colour of the original surface. Fingermarks in blood were also placed directly on the surfaces and treated with one of two enhancement techniques (depending on the colour of the original surface) only (no alginate casting). A comparison of all three methods (alginate casting followed by subsequent enhancement; enhancement of the original surface post-alginate lifting; and enhancement only) was established to determine the most effective technique. The effect of ageing on the enhancement of the fingermark on each surface was investigated using four different ageing periods: $1,7,14$ and 28 days. The sensitivity of each method was assessed through the use of depletion series'.

The enhancement techniques carried out on both the alginate cast and the original surface post-alginate casting were the protein stain acid black 1 (AB1) and the peroxidase reagent leuco crystal violet (LCV). If the original surface was dark in colour, the fluorescent protein stain acid yellow 7 (AY7) was applied instead of AB1. The same methodology was applied for depletion series' treated with the enhancement technique alone (no alginate casting).

\section{Preliminary work}

Preliminary work with the alginate casting material proved that the initial ratio of alginate powder to distilled water needed to be altered from 33.6g of GC Aroma Fine Dust III mixed with $80 \mathrm{~mL}$ distilled water (as per manufacturer guidelines) to $33.6 \mathrm{~g}$ of alginate mixed with $90 \mathrm{~mL}$ distilled water [6]. This ratio provided a better consistency and provided enough material to cast a depletion series of 10 fingermarks in adjacent two columns of five. Furthermore, preliminary work proved the alginate to be ineffective on the non-porous surfaces tested in this experiment (glass and tile) and these were therefore substituted with two new substrates (cardboard and clear plastic bags). 


\section{Preparation of substrates}

Plastic bags, leaflets and 80 gsm white paper were obtained locally from the same source and batch. Square pieces of cardboard were cut from a large cardboard box to ensure all cardboard samples were from the same source. $1 \mathrm{~m}^{2}$ of black polycotton fabric and a pair of dark blue denim jeans were acquired and cut to include in the study. Some substrates (clear plastic bags and leaflets) had areas of different colour (due to patterns and/or writing) and therefore to achieve consistency, the fingermarks were applied to the same sections of the substrate.

\section{Preparation of fingermarks in blood}

$10 \mathrm{~mL}$ of lysed horse blood (at room temperature) was poured into a petri dish. The donor coated their right index finger in the blood before dabbing the finger twice (about two seconds per dab) on chemical free blue paper towel to remove the excess blood. The deposition pressure of each fingermark in blood was not controlled in this study; however, the donor was instructed to attempt to keep a constant pressure in the process. Three replicates of each depletion series were prepared on each substrate for each ageing period. Each depletion series was prepared in two columns: fingermarks 1-5 were planted in the first column followed by fingermarks 6-10 in the adjacent column.

One of these three depletion series was treated with alginate, with the alginate cast being subsequently enhanced with $\mathrm{AB} 1$ and the original post-alginate surface treated with $\mathrm{AB} 1$ (or AY7 if the substrate was dark in colour). The second of these three depletion series was treated with alginate, with the alginate cast being subsequently enhanced with LCV and the original post-alginate surface treated with LCV. The third depletion series was then treated with AB1 directly without the use of alginate (or AY7 if the substrate was dark in colour). This process was then repeated for each of the four ageing periods $(1,7$, 14 and 28 days) and each of the six substrates (black polycotton, denim, cardboard, leaflets, paper, and plastic bags). During each ageing period, the fingermarks in blood were stored on an open bench in the laboratory at room temperature. 


\section{Control samples}

The positive control tests involved treating a depletion series of 10 fingermarks in blood for each ageing period $(1,7,14$ and 28 days) on each of the individual surfaces for each of the techniques (AB1, AY7 and LCV). The negative controls involved applying alginate to each of the substrates where no blood was present on the surface.

\section{Alginate casting}

The alginate was prepared for each required sample by vigorously mixing $33.6 \mathrm{~g}$ of alginate powder to $90 \mathrm{~mL}$ distilled water in a plastic beaker for 30 seconds. The mixture was then applied to the substrate as quickly as possible using a plastic spatula ensuring a consistent layer is produced. A plastic bag was then placed over the top and light pressure was applied (to ensure good contact between the alginate and the substrate). The alginate was then left for two to three minutes to set and dry before removal from the substrate. For paper samples, the alginate had to be removed two minutes after application as longer time periods caused the paper to rip and tear during removal of the cast. All alginate casts were left to dry overnight before chemical treatment.

\section{Preparation and application of enhancement techniques}

\section{Protein stains [7]}

Fixative solution - the fixing solution was necessary to fix the blood marks (prior to applying the protein stain) by precipitation. 5-sulphosalicylic acid dihydrate ( $23 \mathrm{~g}$, Acros) was dissolved in $1 \mathrm{~L}$ of distilled water using a magnetic stirrer. Items to be treated were left in solution for at least five minutes.

Staining solution - the relevant protein stain (AB1 or AY7, $1 \mathrm{~g}, \mathrm{BVDA})$ was stirred for at least 30 minutes in acetic acid $(50 \mathrm{~mL}$, Fisher), ethanol $(250 \mathrm{~mL}$, Fisher) and distilled water $(700 \mathrm{~mL})$ using a magnetic stirrer. Items to be treated were left in solution for at least 10 minutes.

De-staining solution - a solution of acetic acid $(50 \mathrm{~mL}$, Fisher), ethanol (250 mL, Fisher) and distilled water $(700 \mathrm{~mL})$ was used to wash off the excess dye. 


\section{$\underline{\mathrm{LCV}}[8]$}

5-sulphosalicylic dihydrate (10 g, Acros) was dissolved in 3\% hydrogen peroxide $(500 \mathrm{~mL}$, Fisher). Sodium acetate $(3.7 \mathrm{~g}$, Acros) was then added, followed by leuco crystal violet ( $1 \mathrm{~g}$, Acros) and stirred until completely dissolved using a magnetic stirrer. The solution was then transferred to an Ecospray ${ }^{\circledR}$ unit and applied via short bursts of spraying. All samples were left to dry in the fume hood prior to photography and grading.

\section{Photography and Fluorescence}

A Nikon D5100 digital SLR camera with a 18-55mm lens was used to photograph all samples before and after enhancement. Fluorescence observation for AY7 and LCV was performed by means of a Mason Vactron Quaser 2000/30 connected to an Integrated Rapid Imaging System (IRIS) and Crime-lites ${ }^{\circledR}$. For items treated with AY7, fluorescence was observed by exciting with a blue excitation source (band pass filter 385-509 $\mathrm{nm} \mathrm{1 \%}$ cut-on and cut-off points respectively) and viewed with a yellow/orange long pass 510 $\mathrm{nm}$ filter (1\% cut-on point) whereas for items treated with LCV, fluorescence was observed by using a green/yellow excitation source (band pass filter 503-591 nm 1\% cuton and cut-off points respectively) and viewed with a red long pass $593 \mathrm{~nm}$ filter (1\% cut-on point).

\section{Alternative alginate preparation}

Further tests were conducted where the staining solutions (both AB1 and AY7 were tested) were mixed with the alginate powder in the same quantities as a replacement to water. In addition, a water-based protein stain solution was also tested as a replacement to water when mixing with the alginate powder. The recipes for both AB1 and AY7 were altered so that $2 \mathrm{~g}$ of the relevant protein stain (either AB1 or AY7) was stirred for at least 30 minutes in $1 \mathrm{~L}$ distilled water using a magnetic stirrer.

The test was initially performed on clear plastic bags by applying either the alginate/stain mixture or alginate/water-based stain mixture to the fingermarks in blood on paper, black plastic bags and black polycotton. All fingermarks in blood were at least three days old prior to application of the alginate/stain mixture or alginate/water-based stain mixture. The effect of ageing was not considered in this part of the study. 


\section{Grading of fingermarks}

After each method, the following numbered grading system was used in the allocation of grades to the enhanced fingermarks, as suggested by the UK Home Office Centre for Applied Science and Technology (CAST) [9]:

$0=$ No evidence of mark

$1=$ Weak development; evidence of contact but no ridge details

$2=$ Limited development; about one third of ridge details are present but probably cannot be used for identification purposes

3 = Strong development; between one third and two thirds of ridge details; identifiable finger mark

4 = Very strong development; full ridge details; identifiable finger mark

For consistency, the same individuals graded all fingermarks throughout the study. This allowed for a comparison of each fingermark to be made from before enhancement and after enhancement to determine whether the fingermark had positively enhanced (the fingermark grade achieved after enhancement was higher than the grade allocated before enhancement), negatively enhanced (the fingermark grade achieved after enhancement was lower than the grade allocated before enhancement), or displayed no difference (the grade achieved before enhancement was the same as the grade allocated after enhancement). Prior to enhancement, most fingermarks in blood were graded 0 with occasional grades of 1 and very few grades of 2 . 


\section{RESULTS AND DISCUSSION}

\section{Controls}

All positive controls exhibited the expected colour/fluorescent changes in the presence of blood and all negative controls exhibited negative results.

\section{GC Aroma Alginate compatibility}

Preliminary testing for the compatibility of alginate with the chosen substrates (tile, glass, leaflet, paper, polycotton and denim) showed that alginate performed very well on leaflet, paper, polycotton and denim, peeling off easily in one smooth motion. On glass and tile substrates, the alginate demonstrated strong adhesion to the surface and could not be removed without damage to the alginate cast. The alginate had to be scraped from the surface through a levering action between the alginate cast and the surface which was time consuming, difficult and destructive. Several attempts were made to increase the alginate's compatibility with these non-porous surfaces which included: thorough cleaning of both substrates prior to the deposition of blood marks and alginate application; changing the type of water used in the alginate mixture from distilled to tap water and increasing the temperature $\left(40^{\circ} \mathrm{C}, 50^{\circ} \mathrm{C}\right.$ and $\left.60^{\circ} \mathrm{C}\right)$ of the substrates prior to alginate application for varying periods of time ( 5 minutes, 10 minutes, 15 minutes, and 30 minutes). Despite these attempts, there was no difference to the compatibility of the alginate to either glass or tile, with the exception of one attempt where a tile was heated for 10 minutes at $40^{\circ} \mathrm{C}$ prior to alginate application. The results from this one-off success could not be replicated in further trials. Consequently, glass and tile were removed from the list of substrates in the study and replaced with cardboard and clear plastic bags after checking for compatibility with the alginate used in this study. 


\section{Surfaces}

The effectiveness of each surface for alginate casting was assessed by determining the number of identifiable fingermarks (i.e. those graded a " 3 " or "4") on the enhanced alginate casts across all enhancement techniques, ageing periods and depletion series'. The results were poor, with very few identifiable fingermarks on the enhanced alginate casts. Plastic bags yielded alginate casts with the highest number of enhanced identifiable fingermarks; however this was three fingermarks out of the total 80 lifted from plastic bags with alginate followed by enhancement. Furthermore, no grade "4" fingermarks could be achieved using alginate casting followed by subsequent enhancement on any of the surfaces. It is believed that the fabrics (black polycotton and denim) are unable to produce enhanced fingermarks in blood on alginate casts of sufficient quality due to the weave pattern, which appeared to be more pronounced in denim (figure 1c). This is in contrast to the successful recovery of footwear impressions in blood on fabrics followed by subsequent enhancement $[3,5,6]$. Polycotton could not produce an enhanced fingermark on an alginate cast above a grade " 2 ", and denim could not produce an enhanced fingermark on an alginate cast above a grade "1". Figure 1 provides examples of fingermarks lifted by alginate casts followed by treatment with AB1 or LCV. Figure $1 \mathrm{~b}$ and $\mathrm{c}$ show fingermarks treated with LCV which appear to be of lesser quality when compared to those treated with AB1.

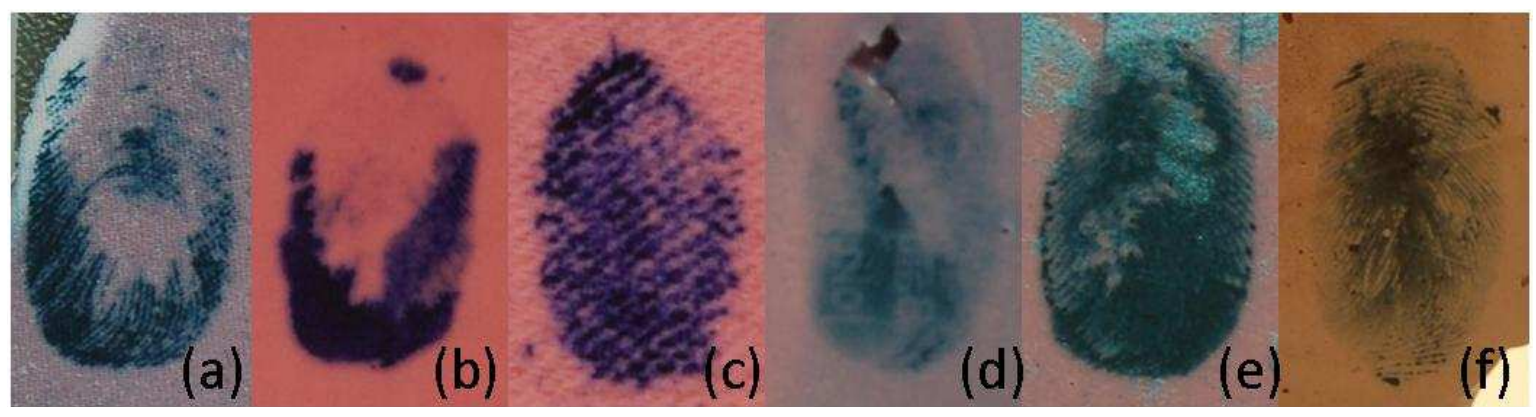

Figure 1: Examples of lifted alginate casts followed by chemical treatment from different surfaces: (a) polycotton; (b) cardboard; (c) denim; (d) leaflet; (e) paper; and (f) plastic bag 


\section{Enhancement techniques - enhancement on alginate cast}

The results demonstrated that $\mathrm{AB} 1$ yielded the highest number of positive enhancements (with a total of 51 positive enhancements compared with the 33 positive enhancements achieved by LCV). In general, the results for those fingermarks in blood lifted with alginate and subsequently treated with $\mathrm{AB} 1$ were sharper and provided more defined detail whereas those casts subsequently treated with LCV demonstrated a high degree of diffusion. This appears to be in line with other studies $[3,6]$ where AB1 was also found to provide superior enhancement to LCV in the enhancement of footwear marks in blood on GC Aroma alginate casts. In addition, it should be noted that the highest grade allocated to a fingermark in blood on an alginate cast enhanced with $\mathrm{AB} 1$ was a grade "3", whereas that achieved through LCV enhancement was a grade "1".

\section{Enhancement techniques - enhancement on original blood mark post-alginate lifting}

Similar to alginate casting followed by chemical enhancement, AB1 yielded the highest percentage of positive enhancements (with $28 \%$ positive enhancements, compared to $8 \%$ using AY7 and 18\% using LCV). Figure 2 illustrates the difference in fingermark quality between the three enhancement techniques on the original post-alginate surface. It was observed that the fluorescence of marks treated with AY7 appeared to be poor on all surfaces.

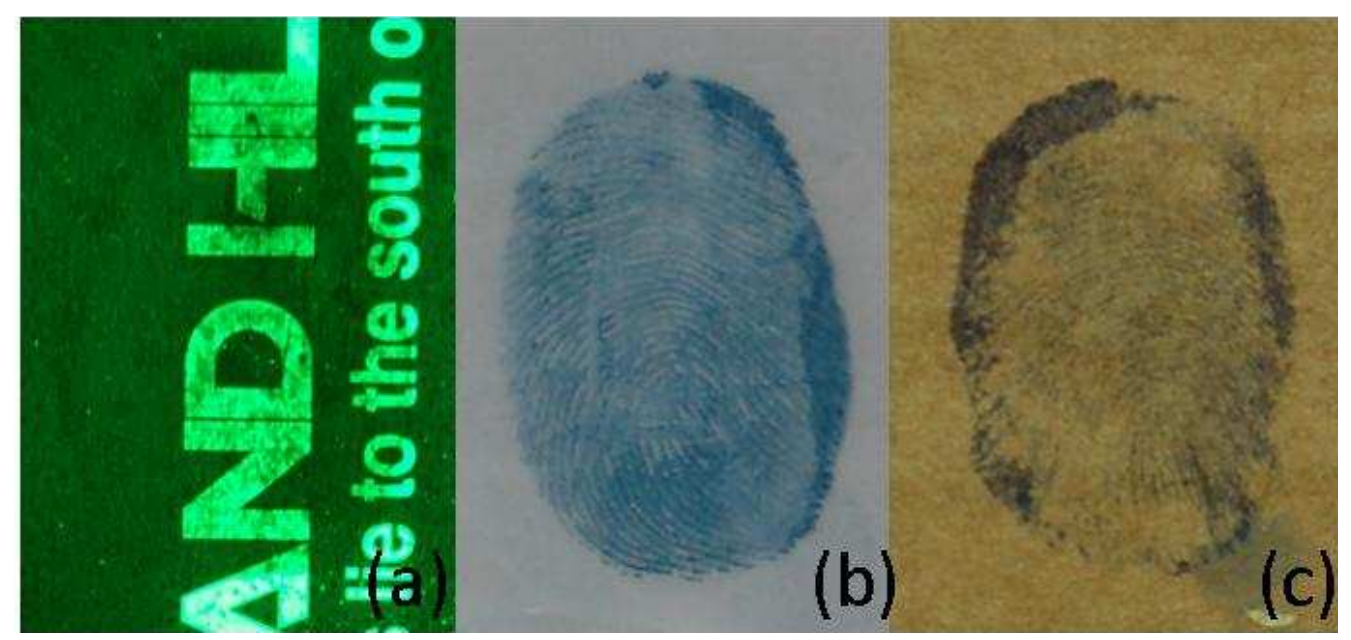

Figure 2: Enhancement of fingermarks in blood on different post-alginate surfaces with (a) AY7 fluorescence (7 days on leaflet, no. 2 in the series; (b) AB1 (14 days on plastic bag, no. 8 in the series); (c) LCV (28 days on cardboard, no. 2 in the series) 
Enhancement techniques - enhancement on substrate without the use of alginate

The results for this part of the study demonstrated that $\mathrm{AB} 1$ is the most effective enhancement technique since it yielded the highest number of positive enhancements. Figure 3 demonstrates the use of $\mathrm{AB} 1$ enhancement without the use of alginates. In general, AY7 fluorescence appeared to be poor across all surfaces and the ridge detail was inferior to that obtained by AB1. Preliminary work, in line with other studies [3], revealed that alginate lifting of marks treated with chemicals was limited.

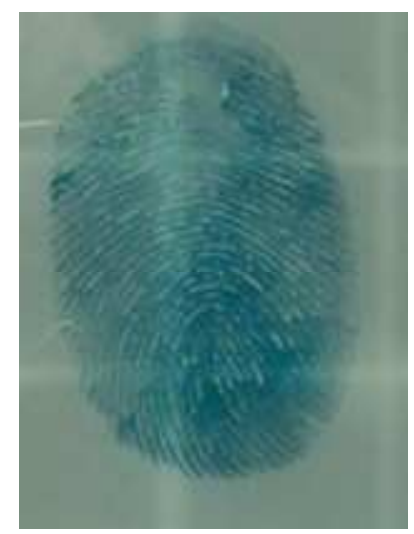

Figure 3: Enhancement of a fingermark in blood on a plastic bag without the use of alginate with AB1 (28 days, no. 7 in the series)

\section{Ageing of fingermarks in blood}

The data from each of the enhancement methods (enhanced alginate casts; enhancement of the original surface post-alginate lifting; and enhancement only) was analysed to determine whether there was any correlation between the age of the fingermarks in blood and the quality of the enhanced fingermark. The number of enhanced marks graded 0,1 , 2, 3 and 4 was determined for each individual ageing period (1, 7, 14 and 28 days) and for each enhancement method (including all enhancement techniques utilised in each enhancement method). Figure 4 demonstrates a similar pattern of grading in the enhanced fingermarks in blood on the alginate casts across the different ageing periods in this study. It should be noted that, prior to enhancement, most fingermarks in blood were graded 0 with occasional grades of 1 and very few grades of 2. Figure 4 represents the final grade given to the enhanced fingermark in blood. The number of marks graded 2, 3 and 4 appeared to reduce with increasing ageing periods. 


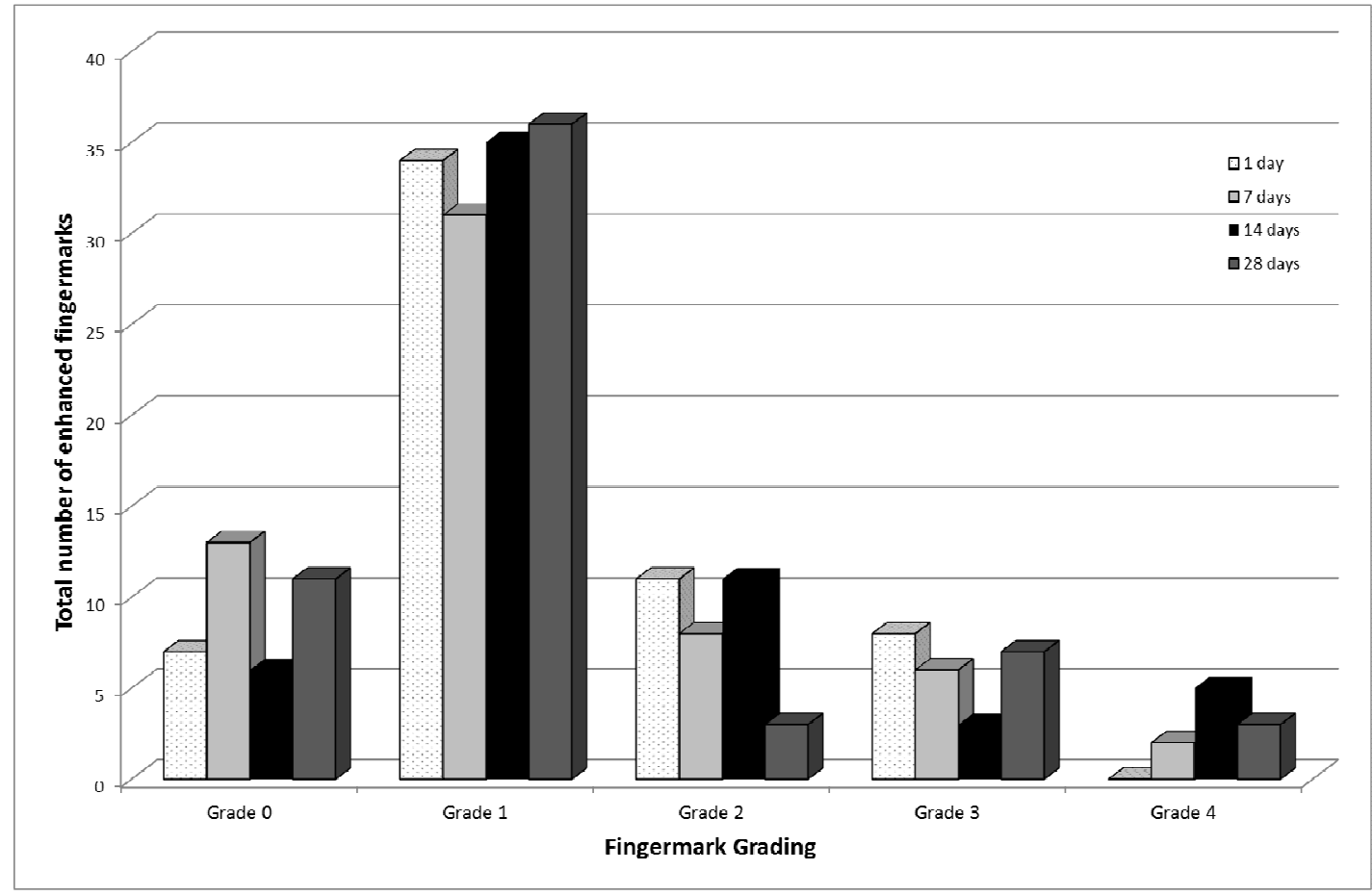

Figure 4 - Comparison of fingermark grading in the study across different ageing periods

There was a limited degree of correlation between the age of the fingermark in blood and the quality of the enhanced fingermark on the post-alginate surface. The number of grade "0" fingermarks decreased with the age of the fingermark and in general there was an increase in grade 2, 3, and 4 fingermarks as the ageing period increased, although the trend was not completely linear. The ageing period of 28 days provided the highest number of identifiable (grade 3 and 4) fingermarks, and the lowest number of grade 0 fingermarks. Further results demonstrated no correlation between the age of the fingermark in blood and the quality of the enhanced fingermark on the surface treated by enhancement techniques without the use of alginate. 


\section{Depletion Series}

A degree of correlation was observed between the amount of blood on the fingermark and the enhanced fingermark quality across the different enhancement methods. The number of grade 0 fingermarks increased down the depletion series and the number of grade 2 and 3 fingermarks was more abundant in the middle of the depletion series (figure 5). This may be explained by the fact that marks at the beginning of the depletion series are smudged by excess blood and do not demonstrate ridge detail, whereas those fingermarks at the end of the depletion series do not contain enough blood on the ridges to be enhanced. It should be noted that, prior to enhancement, most fingermarks in blood were graded 0 with occasional grades of 1 and very few grades of 2. Figure 5 represents the final grade given to the enhanced fingermark in blood.

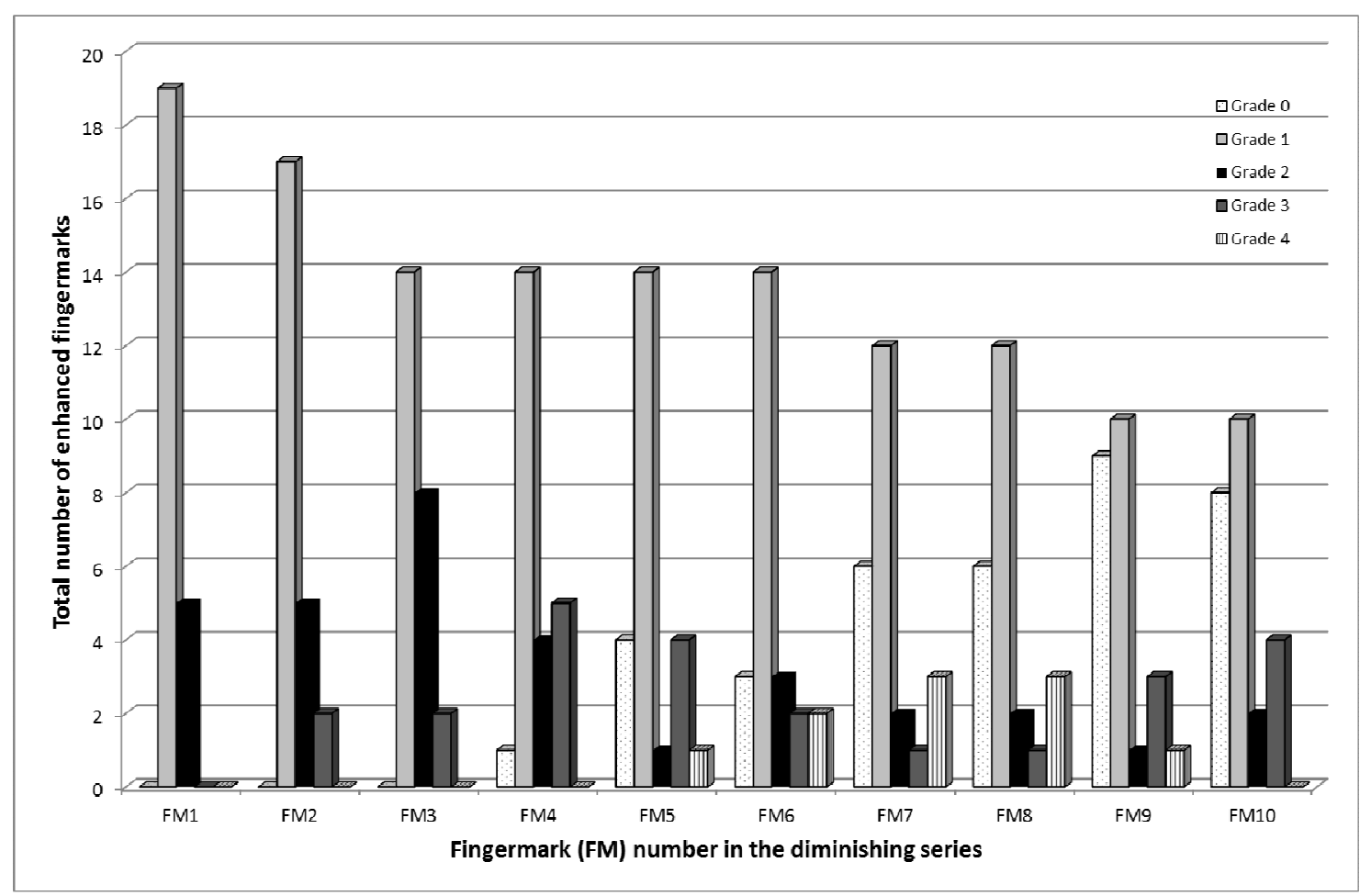

Figure 5: Comparison of fingermark grading across the diminishing series 


\section{Relationship between enhancement method and fingermark quality}

This study investigated whether the use of alginate on fingermarks in blood provides superior enhancement compared to enhancement alone (i.e. no alginate casting). The results obtained suggest that if alginate casting is to be used, then chemical treatment should be done on the alginate cast once lifted rather than chemical treatment on the substrate followed by alginate lifting. The results in thus study also demonstrate that the use of alginate to lift fingermarks in blood with subsequent enhancement on the cast is inferior to the direct application of enhancement techniques to the substrate. The highest percentage of positive enhancements originated from those samples treated with $A B 1$ enhancement only (with a total of $43 \%$ of fingermarks being positively enhanced, compared to $28 \%$ of fingermarks on the original surface post-alginate lifting, and $21 \%$ of fingermarks on the alginate casts). Figure 6 illustrates the difference in fingermark quality between each of the enhancement methods (representing the highest grade achieved by each method).

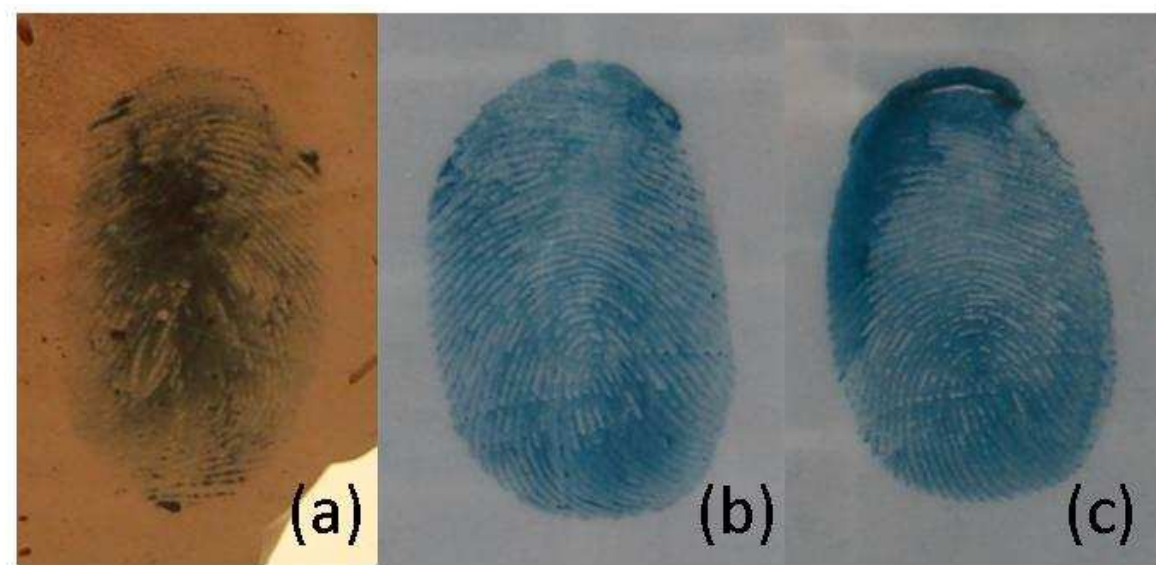

Figure 6: Enhancement of fingermarks in blood on a plastic bag with (a) alginate cast treated with AB1 (1 day, no. 5 in the series; (b) AB1 treatment on original surface postalginate lifting (14 days, no. 7 in the series); (c) AB1 enhancement without the use of alginate (14 days, no. 7 in the series)

\section{Further work - alternative alginate preparation}

The first alternative preparation involved the same quantities of alginate powder and liquid as described previously; however, a staining solution of AB1 or AY7 (700 mL water, $250 \mathrm{~mL}$ ethanol, $50 \mathrm{~mL}$ acetic acid) was used rather than water. This mixture was 
a fluid liquid (as opposed to the relatively stiff properties the alginate-water mixture demonstrates) that would not set after application onto the fingermarks in blood, even after 15 minutes (alginate usually takes 2-3 minutes to set). It did, however, enhance the fingermarks in blood on the original surface.

A second alternative preparation was conducted where an additional scoop of alginate powder $(8.4 \mathrm{~g})$ was mixed with the same volume of staining solution (AB1 or AY7). This created a grainy, dry paste where some of the alginate had not mixed properly. After application onto fingermarks in blood on the plastic bags, the mixture did not set, even after 10 minutes and still displayed malleable properties. The non-setting properties of alginate using the staining mixture may be due to the acetic acid and/or ethanol in solution.

A third alternative preparation was prepared by mixing the same ratios of power to liquid (as described in the Material and Method section) and using a water-based protein stain solution rather than water for mixing. Water-based protein stain solution are prepared by dissolving $2 \mathrm{~g}$ of the required stain in $1000 \mathrm{~mL}$ distilled water with the addition of $20 \mathrm{~g}$ citric acid, however, in this study citric acid was not used as the acid may interfere with the alginate properties. Initial work with AY7 did not yield suitable enhancement and work focused mainly on AB1. This third preparation was adopted for further work since it appeared to provide a similar consistency of alginate when mixed with water. 10 mark depletion series' of fingermarks in blood were prepared on four surfaces and left to age overnight: clear plastic bags; white paper; black plastic bags; and black polycotton. The preparation allowed the lifting and enhancement of fingermarks in blood on the cast (figure 7) as well as enhancement on the original surface (figure 8) in one step. Fingermarks visualised on casts are a mirror image of the original mark. AB1 did not provide direct enhancement on black plastic bags due to poor contrast and the use of fluorescence and AY7 did not aid to improve the contrast on dark plastic bags. The alginate casts were rinsed after setting to remove excess dye from the background and allow for better contrast between the enhanced fingermark and the background. In general, the original surface did not require additional rinsing to remove the excess dye. 

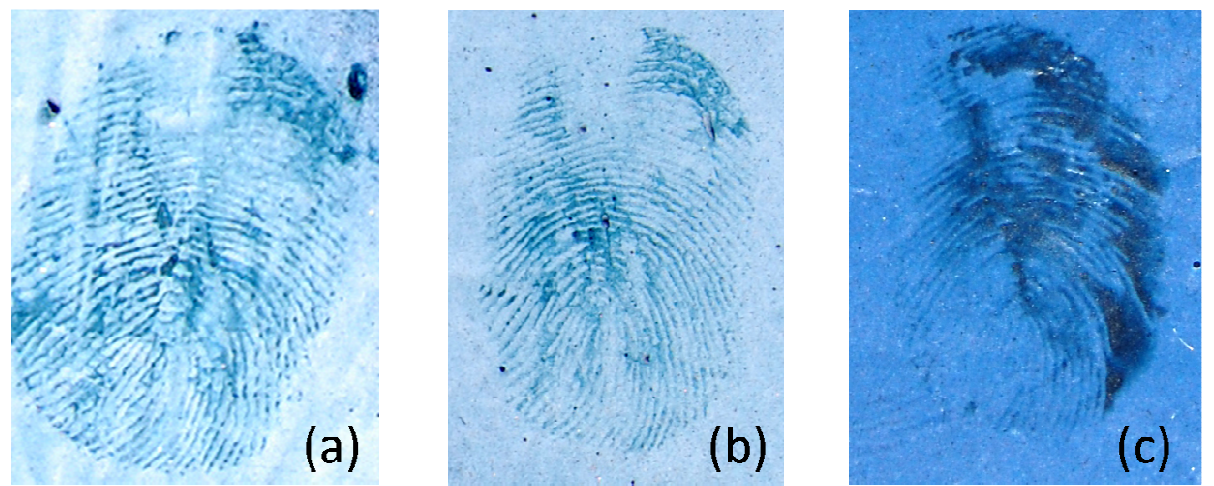

Figure 7: Enhancement of fingermarks in blood on clear plastic bags lifted with alginate casts prepared using the alginate/water based $\mathrm{AB} 1$ stain mixture
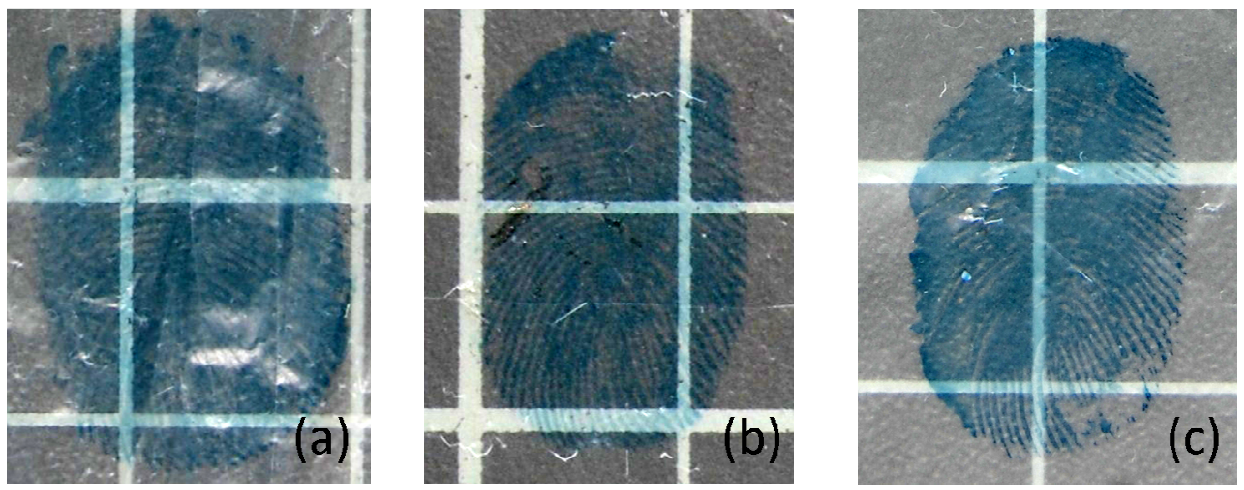

Figure 8 - Enhancement of fingermarks in blood on clear plastic bags when in contact with alginate prepared using the alginate/water based $\mathrm{AB} 1$ stain mixture (corresponding to figure 7)

The use of leuco crystal violet (LCV) mixed with alginate was not considered in this study, mainly due to the fact that poor enhancement was obtained when LCV was applied post-alginate lifting. Furthermore, a separate study [3] assessed the mixing of LCV with alginate but the results obtained were not successful due to the acidic nature of the LCV solution interfering with the alginate casting. The results discussed for the alternative preparations in this study are preliminary and further work is required into the reproducibility of the use of the alginate/stain mixture, as well as an investigation into its performance on a variety of different surface types and aged fingermarks in blood. 


\section{CONCLUSION}

In this study, it was concluded that treating the fingermarks in blood with AB1 without the use of alginate provides a higher number of positively enhanced fingermarks than using alginate. It is hypothesised that the fine detail of fingermarks in blood is not successfully lifted and subsequently enhanced. Moreover, the results were not consistent when occasionally the alginate lifted and enhanced the fingermark in blood to provide an identifiable fingermark.

Preliminary work involving the mixing of the alginate powder with water-based staining solutions appears to provide superior enhancement when compared to the conventional alginate mixture of alginate and water. This technique might be suitable for when direct treatment on the substrate is not suitable due to interference from the background of the substrate (lack of contrast) or background fluorescence. Additionally, its use for the treatment of vertical surfaces and emulsion paints may solve issues associated with vertical diffusion of blood marks with techniques such as luminol as well as avoid contamination of the scene with chemicals and solvents. Further work using a split depletion series for a comparison of fingermarks in blood pre- and post-alginate treatment as well as using water-based protein stain solutions and alginate is required to understand the benefits and mechanisms of this alternative preparation. 


\section{REFERENCES}

1. Inoue, K., Song, Y.X., Fujii, K., Kadokawa, A. And Kanie, T. 1999. Consistency of alginate impression materials and their evaluation. Journal of Oral Rehabilitation. 26(3): pp.203-207.

2. McCabe, J.F. and Walls, A.W.G. 2008. Applied dental materials. $9^{\text {th }}$ ed. Oxford: Blackwell Publishing Ltd. pp. 158.

3. Wiesner, S., Izraeli, E., Shor, Y., and Domb, A. 2013. Lifting bloody footwear impressions using alginate casts followed by chemical enhancement. Journal of Forensic Sciences. 58(3): pp.782-788.

4. Adair, T.W. 2005. Casting two-dimensional bloody shoe prints from concrete, fabric, and human skin: A review of several methods with recommendations. Journal of Bloodstain Pattern Analysis. March: pp. 4-8.

5. Wiesner, S., Izraeli, E., and Shor, Y. 2007. Lifting and enhancing bloody footwear impressions on clothing. European Meeting for Sp/TM Examiners. Denmark: Copenhagen.

6. Farrugia, K.J., NicDaéid, N., Savage, K.A., and Bandey, H. 2010. Chemical enhancement of footwear impressions in blood deposited on fabric - evaluating the use of alginate casting materials followed by chemical enhancement. Science and Justice. 50(4): pp.200-204.

7. Bowman, V. ed. 2004. Manual of fingerprint development techniques. $2^{\text {nd }}$ ed. London: U.K. Home Office Scientific Development Branch. pp. 105-177.

8. Bodziak, W.J. 1996. Use of leuco crystal violet to enhance shoe prints in blood. Forensic Science International. 82(1): pp. 45-52.

9. Sears, V.G., Bleay, S.M, Bandey, H.L., and Bowman, V.J. 2012. A methodology for finger mark research. Science and Justice. 52(3): pp.145-160. 